\title{
ARTICLE
}

\section{Clinical coding and data quality in oculoplastic procedures}

\author{
Valerie Juniat $^{1} \cdot$ Sarju Athwal $\mathbb{B}^{1,2} \cdot$ Mona Khandwala ${ }^{1}$
}

Received: 30 July 2018 / Revised: 24 February 2019 / Accepted: 13 March 2019 / Published online: 3 June 2019

(c) The Royal College of Ophthalmologists 2019

\begin{abstract}
Introduction Hospitals in England are reimbursed via national tariffs set out by NHS England. The tariffs payable to hospitals are determined by the activity coded for each patient's hospital visit. There are no national standards or publications within oculoplastics for coding accuracy. Our audit aimed to determine the accuracy of coding oculoplastic procedures carried out in theatres and to assess the financial implications of any discrepancies.

Methods We carried out a prospective audit of consecutive oculoplastic procedures performed at one hospital site over a 6-week period. We subsequently created a coding proforma and performed a re-audit using the same methods.

Results In the first cycle, clinical coding was 'correct' in $30.7 \%$ of cases, 'incomplete' for $12.9 \%$ and 'incorrect' for $56.5 \%$. Of the 'incorrect' codes, $54.3 \%$ were coded as non-oculoplastic procedures (e.g. extraocular muscle surgery). We discussed our findings with the coding team in order to address the sources of error. We also created a 'tick box' coding proforma, for completion by surgeons. Our re-audit results showed an improvement of 'correct' coding to $85.7 \%$.

Conclusion Clinical coding is complex and vulnerable to inaccuracy. Our audit showed a high rate of coding error, which improved following collaboration with our coding team to address the sources of error and by creating a coding proforma to improve accuracy. Accurate clinical coding has financial implications for hospital trusts and consequently Clinical Commissioning Groups. In times of severe financial pressures, this could be a valuable tool, if rolled out over all specialities, to make much needed savings.
\end{abstract}

\section{Introduction}

The structure of NHS finances and reimbursement is complex. The introduction of Payment by Results (PbR) into the NHS in 2004 replaced block contract payments where hospitals received an annual sum to provide broadly specified services [1,2]. This led to hospitals receiving payment for individual patient episodes based on a national tariff. The tariffs actually payable to hospitals are determined by the activity coded for each patient's hospital visit. Uncoded activity is not reimbursed, and this has financial implications.

Valerie Juniat

vjuniat@doctors.org.uk

1 Maidstone and Tunbridge Wells NHS Trust, Hermitage Lane, Maidstone, Kent ME16 9QQ, UK

2 Moorfields Eye Hospital NHS Trust, 162 City Road, London EC1V 2PD, UK

\section{Overview of clinical coding}

Clinical coding involves the translation of patients' clinical diagnoses and procedures into an internationally recognised, coded language [2, 3]. Clinical diagnoses are assigned using the World Health Organisation's International Statistical Classification of Diseases and Other Health Problems (ICD) codes, of which the latest revision is ICD-10 [4]. NHS procedures are coded using version 4.4 of the Office of Population, Censuses and Surveys Classification of Surgical Operations and Procedures (OPCS-4) system [5].

For patients admitted for surgical interventions, their clinical diagnoses and procedures are coded to generate payment. Certain oculoplastics procedures have specific codes (e.g. evisceration) but other procedures would fall under more generic codes (e.g. inferior retractor plication under entropion correction).

The ICD-10 and OPCS-4 codes are entered into a computer software called the HRG4+ Local Payment Grouper [6], used throughout the UK, to generate Healthcare Resource Group (HRG) codes for that patient 
episode. The first four digits of the HRG are known as the HRG root and describe the disorder or treatment, such as BZ44A 'Major Oculoplastics Procedures, 19 years and over, with complication or comorbidities (CC) score $2+$ '. In many HRGs the fifth character indicates the level of $\mathrm{CC}$ of an HRG, which is automatically calculated by the HRG software. The number of diagnoses coded, such as diabetes, hypertension, and even concurrent anticoagulant usage, therefore has an impact on the CC score and subsequent HRG. Additionally, the order of the diagnoses does not impact on the HRG code, whereas the order of the procedures does.

The HRG codes are subsequently assigned a unit of payment, the price of which is fixed in relation to an annual national tariff [7]. This tariff is calculated through a process known as 'reference costing' by averaging the unit cost of providing defined services to NHS patients [6, 8-10]. The final payment is further weighted by the type of admission (elective or emergency), whether it is a paediatric case, length of stay and ITU admission, and by the 'Market Forces Factor' - extra renumeration given to reflect the cost of living in the area where the service is provided [3, 9]. Coding errors may therefore also have financial implications for the Trust.

Clinical coding is complex and lends itself to being vulnerable to inaccuracy. In the UK, trained non-medical personnel perform clinical coding. Often, multiple departments are involved in the patient's coding journey. In our Trust, the theatre team and the clinical coding team input the OPCS-4 codes using the details from the handwritten operation notes. The clinical coding team also uses the whole patient record to obtain data regarding clinical diagnoses, but they do not have access to theatre coding. Surgeons do not have a role in data input. Subsequent financial reimbursements rely solely on the clinical coding team's input.

\section{Purpose of study}

There is no national standard for coding accuracy. A systematic review of clinical coding found an overall median accuracy of $83.2 \%$, with higher procedure accuracy at $84.2 \%$ compared with diagnostic accuracy at $80.3 \%$ [11]. The review revealed large variation in accuracy rates between studies ranging from 50.5 to $97.8 \%$.

A study of clinical coding accuracy across all surgical specialties found at least one change to the original coding in $51 \%$ of cases, with $13 \%$ of these being changes to primary diagnoses and $12 \%$ to primary procedures [12]. The highest proportion of changed primary diagnoses and procedures were seen in Ophthalmology, with an overall change of $22 \%$, leading to a changed HRG coding in $10.1 \%$ and an income variance of $£ 25.19$ per patient.
The main aim of our study was to determine the accuracy of coding oculoplastic procedures. We also wished to assess the financial implications of any discrepancies. To our knowledge, this is the first publication on coding accuracy within oculoplastics.

\section{Methods}

We prospectively collected and analysed data for all consecutive oculoplastic procedures carried out at one hospital site over a 6 -week period from $2 / 10 / 17$ to $16 / 11 / 17$. Patient demographics and clinical diagnoses were collected from clinical notes. Operation details were recorded on the audit proforma by one of the operating surgeons at the time of surgery. Theatre coding applied by our staff was obtained from theatre coding software (TheatreMan ${ }^{\mathrm{TM}}$; Trisoft, Peterborough, United Kingdom). Clinical coding applied by our staff was obtained from clinical coding software (Medicode $^{\mathrm{TM}}$ Clinical Encoder; 3M, Bracknell, United Kingdom).

We identified the correct ICD-10 codes for the primary and secondary clinical diagnoses (including morbidities and post-procedural complications) from the international, standardised handbook on ICD-10. We identified the correct procedure list for each patient based on the recorded surgical information and the authors' knowledge of which surgical procedures each patient underwent. We then identified the OPCS-4 code for the primary and secondary procedures performed from the national, standardised OPCS handbook. Finally, we checked the accuracy of the theatre codes (OPCS-4) and initial clinical codes (ICD-10 and OPCS-4) applied by our staff against the correct ICD-10 and OPCS-4 codes we had identified.

We classified the results as 'correct', 'incomplete' or 'incorrect'. 'Incomplete' cases were those where the type of anaesthetic was not included. 'Incorrect' cases included wrong procedure, data entry of an additional but incorrect procedure, or where an additional procedure had been carried out but was omitted. Where we identified an apparent error in the initial coding, we discussed the cases further with experienced clinical coders. The coding was changed if the clinical coders agreed with our assessment. A similar method has been published elsewhere [2].

We used the HRG software to generate the HRG tariffs associated with each procedure using the initial procedural codes and the revised codes. Financial estimates are based on the latest available hospital tariffs for 2017-2018. Any resulting income variance from the revised codes and HRGs were recorded. We analysed the data using Excel (Microsoft; Redmond, Washington, United States).

Following our first cycle, we created a tick box coding proforma in collaboration with the oculoplastic consultants, 


\section{OCULOPLASTICS SURGICAL CODING FORM \\ (Tick all that apply)}

\begin{tabular}{|c|c|c|}
\hline PTOSIS/BLEPHAROPLASTY & CODE & $\sqrt{ }$ \\
\hline Blepharoplasty of upper eyelid & C13.2 & \\
\hline Levator muscle technique (ALR) & C18.1 & \\
\hline Frontalis muscle support operation (silicone) & C18.2 & \\
\hline Correction of ptosis using sling of fascia & C18.3 & \\
\hline Tarsomullerectomy & C18.5 & \\
\hline Correction of ptosis by aponeurotic repair & C18.6 & \\
\hline LACRIMAL & CODE & $\sqrt{ }$ \\
\hline Canaliculodacryocystorhinostomy & C25.1 & \\
\hline Dacryocystorhinostomy and insertion of tube & C25.3 & \\
\hline Revision of anastomosis between lacrimal sac and nose & C25.5 & \\
\hline Drainage of nasolacrimal duct (inc. lacrimal/canalicular stent) & C27.1 & \\
\hline Irrigation of nasolacrimal duct & C27.3 & \\
\hline Probing of nasolacrimal duct (+/- endoscopic-assisted) & C27.5 & \\
\hline Repair of lacrimal canaliculus & C29.1 & \\
\hline Enlargement of lacrimal punctum ( 3 snip, punctoplasty) & C29.2 & \\
\hline Functional endoscopic nasal surgery & Y76.2 & \\
\hline
\end{tabular}

\begin{tabular}{|l|l|l|}
\hline TARSORRHAPHY & CODE & $\mathbf{v}$ \\
\hline Central tarsorrhaphy & C16.1 & \\
\hline Lateral tarsorrhaphy & C16.2 & \\
\hline Medial tarsorrhaphy & C16.3 & \\
\hline Revision of tarsorrhaphy & C16.5 & \\
\hline Complete protective suture of eyelid (+ Frost suture) & $\mathrm{C} 20.1$ & \\
\hline
\end{tabular}

Complete protective suture of eyelid (+ Frost suture)

\begin{tabular}{|l|l|l|}
\hline TRICHIASIS & CODE & V \\
\hline Correction of trichiasis & C15.3 & \\
\hline Epilation of eyelash & C22.6 & \\
\hline Excision of lash follicles & C22.8 & \\
\hline
\end{tabular}

Date:

Name:

VJ/SA v1.1 13/02/2018

Fig. 1 Oculoplastics surgical coding form

theatre staff and the coding team (Fig. 1). We then prospectively collected and analysed data for all consecutive oculoplastic procedures carried out at one hospital site over a 6-week period from $9 / 4 / 18$ to $17 / 5 / 18$ as a re-audit using the same method.

\section{Results}

\section{Demographics}

\section{First cycle}

Sixty-five oculoplastic procedures were performed during this 6-week period. Three procedures were excluded as they had not been coded by the clinical coding department at the time of data analysis, leaving 62 procedures for analysis.

The median age of the patients was 70 years old (range: 4-90). Thirteen patients had bilateral surgery, 25 had left eye surgery, and 24 had right eye surgery. Twelve patients had general anaesthetic, 22 patients had local anaesthetic with sedation, and 28 patients had local anaesthetic alone.
Maidstone and Tunbridge Wells WHS

NHS Trust

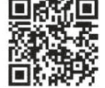

\begin{tabular}{|c|c|c|c|}
\hline LID MALPOSITION & & CODE & $\mathbf{v}$ \\
\hline Replacement of lateral canthal tendon usin & tarsal strip & C09.1 & \\
\hline Lateral canthal sling (canthopexy) & & C11.8 & \\
\hline Correction of ectropion (including medial ss & ndle) & C15.1 & \\
\hline Correction of entropion & & C15.2 & \\
\hline Correction of cicatricial ectropion & & C15.4 & \\
\hline Correction of cicatricial entropion & & C15.5 & \\
\hline LESION REMOVAL/RECONSTRUCTION & & CODE & $\mathbf{v}$ \\
\hline Excision of lesion of eyebrow & & C10.1 & \\
\hline Suture of eyebrow & & C10.4 & \\
\hline Incision of lesion of eyebrow & & C10.5 & \\
\hline Excision of lesion of canthus & & C11.1 & \\
\hline Graft of skin to canthus & & C11.5 & \\
\hline Repair of medial/lateral canthus (including & ees) & C11.8 & \\
\hline Excision of lesion of eyelid & & C12.1 & \\
\hline Incision/curettage of lesion of eyelid & & C12.4 & \\
\hline Wedge excision of lesion of eyelid & & C12.6 & \\
\hline Flap of skin to eyelid & & C14.1 & \\
\hline Graft of skin to eyelid & & C14.2 & \\
\hline Anterior lamellar reconstruction of eyelid & Tick both for full & C14.8 & \\
\hline Posterior lamellar reconstruction of eyelid & & C14.8 & \\
\hline Suture of eyelid (repair of laceration) & & C17.1 & \\
\hline Drainage of lesion of eyelid & & C19.1 & \\
\hline Biopsy of lesion of eyelid & & C22.2 & \\
\hline
\end{tabular}

ADDITIONAL PROCEDURES: add any procedures not covered above

Patient

There were two emergency cases (one evisceration, one temporal artery biopsy), with the remainder being elective procedures.

The most commonly performed procedures were lateral tarsal strip combined with an associated procedure (16\%), blepharoplasty (10\%), ptosis repair (10\%), and lesion excision with biopsy (10\%). A full breakdown of the procedures is shown in Fig. 2.

\section{Second cycle}

Forty-two oculoplastic procedures were performed during the re-audit period. No procedures were excluded.

The median age of the patients was 71 years old (range: 3-90). Nine patients had bilateral surgery, 21 had left eye surgery, and 12 had right eye surgery. Nine patients had general anaesthetic, 13 patients had local anaesthetic with sedation, and 20 patients had local anaesthetic alone. All cases were elective surgery.

The most commonly performed procedures were basal cell carcinoma excision and reconstruction $(28.6 \%)$ and dacryocystorhinostomy (9.5\%). Further breakdown of the procedures is shown in Fig. 3. 
Fig. 2 Overview of oculoplastic procedures carried out during first cycle
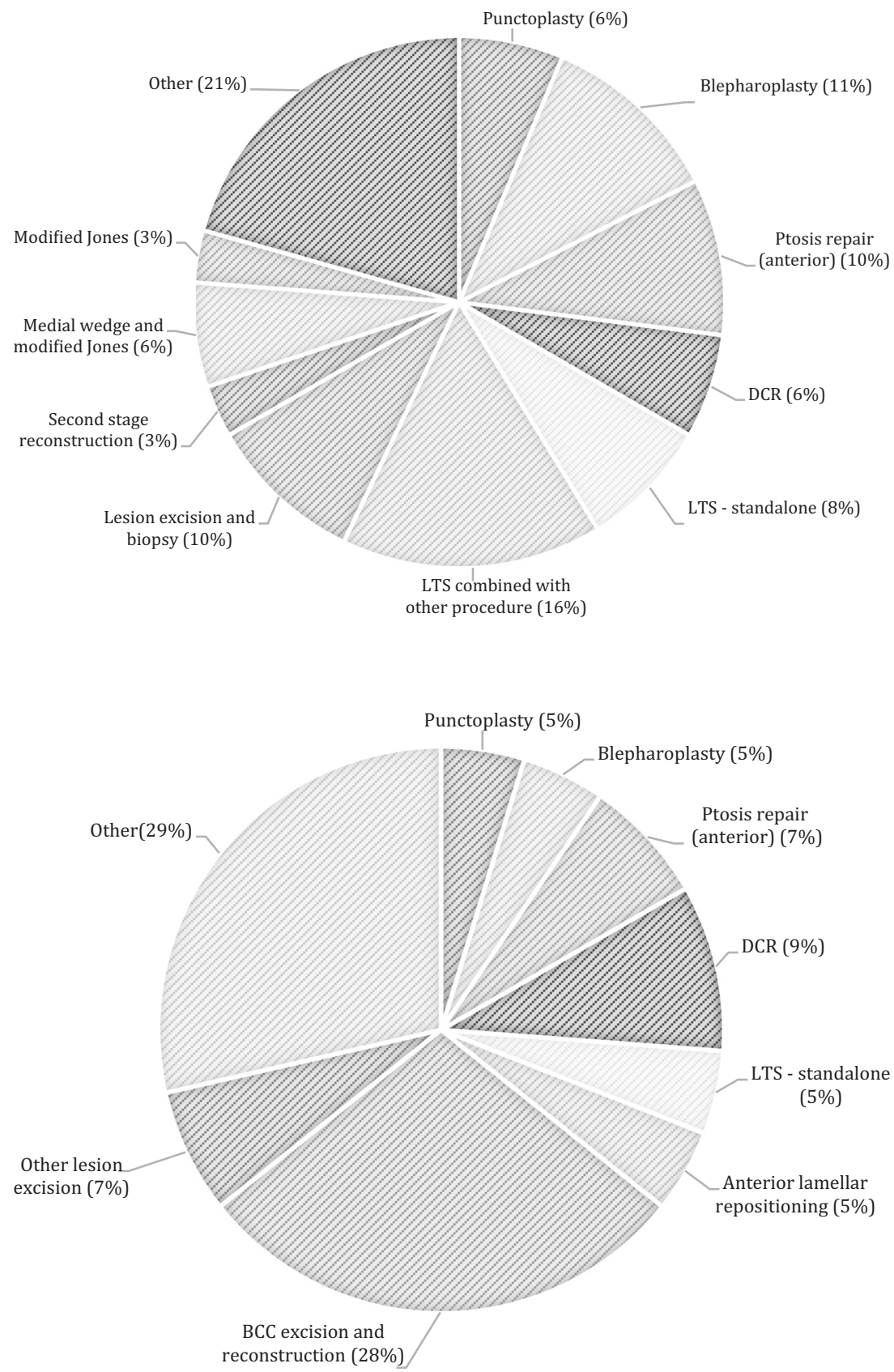

Fig. 3 Overview of oculoplastic procedures carried out during re-audit cycle

\section{Accuracy of coding}

The standard of our audit for procedural coding accuracy was $84.2 \%$, as taken from Burns et al.'s systematic review [11]. The results of our first and re-audit cycles are summarised in Table 1.

In our first cycle for clinical coding, the accuracy rate for our theatre coding was $48.4 \%$ and clinical coding was $30.7 \%$. Data were 'incomplete' for $12.9 \%$ of cases and 'incorrect' for $56.5 \%$. $100 \%$ of procedures with 'incomplete' classification were due to failure of coding the anaesthetic.
Of the procedures that were classified as 'incorrect', $54.3 \%$ were coded as non-oculoplastic procedures (extraocular muscle surgery or 'repair and suture of organs'), $25.7 \%$ were coded with the wrong oculoplastic procedure (e.g. ectropion instead of entropion correction), $25.7 \%$ had an additional, incorrect procedure coded e.g. coding of a 'pedical flap' in graft lid reconstruction, and $28.6 \%$ omitted an additional procedure that had been carried out (e.g. by not recording the biopsy as part of eyelid tumour excision). $22.9 \%$ had more than one source of error. 
Table 1 Accuracy of coding procedures

\begin{tabular}{|c|c|c|c|c|c|}
\hline & \multirow[b]{2}{*}{ Standard } & \multicolumn{2}{|l|}{ Theatre } & \multicolumn{2}{|l|}{ Clinical coding } \\
\hline & & First cycle (62) & Re-audit cycle (42) & First cycle $(62)$ & Re-audit cycle (42) \\
\hline Correct & $84.2 \%$ & $48.4 \%(30)$ & $59.5 \%(25)$ & $30.7 \%(19)$ & $85.7 \%(36)$ \\
\hline Incomplete & - & $35.5 \%(22)$ & $9.5 \%(4)$ & $16.1 \%(10)$ & $0 \%$ \\
\hline Incorrect & - & $16.1 \%(10)$ & $31 \%(13)$ & $53.2 \%(33)$ & $14.3 \%(6)$ \\
\hline
\end{tabular}

We discussed the findings with our clinical coding team to address the sources of error. We discovered that their main sources of difficulties included deciphering surgeons' handwriting, interpreting operation notes where there was significant amount of detail, and understanding muscle nomenclature. Of note, our coders were interpreting and coding orbicularis oculi muscle as an extraocular muscle.

Following our first cycle, we created a coding proforma in collaboration with other relevant clinicians, theatre staff and coding staff. The operating surgeon subsequently filled in the proforma in theatre for every oculoplastic case during the re-audit cycle. Our re-audit demonstrated an improvement in coding accuracy, rising from 48.4 to $59.5 \%$ for theatre coding, and 30.7 to $85.7 \%$ for clinical coding.

\section{Financial implications-first cycle}

We assessed the financial impact of coding inaccuracies. Accurate coding resulted in HRG codes associated with higher tariffs for eight cases (12.9\%).

The incorrect extraocular muscle surgery coding had generated an estimated $£ 4606$ more income than the auditors' coding. However, the auditors generated a net increase of $£ 473$ during the audit period through removal of the incorrect extraocular muscle surgery data and accurately coding all other cases.

Finally, the type of anaesthetic did not change the overall HRG code.

\section{Discussion}

\section{Main findings}

Our audit showed a high rate of procedural coding error in the first cycle, with a higher rate of incorrect coding in clinical coding $(56.5 \%)$ compared with theatre coding $(16.1 \%)$. However, there were no errors in coding diagnoses. As set out above, $54 \%$ of procedural coding error was due to erroneously coding our oculoplastic procedures as extraocular muscle surgery or 'repair and suture of organs', which changed the overall HRG tariff. Other sources of error include wrong procedure, additional but incorrect procedure, and not coding an additional procedure. In 6 weeks, we estimate that accurate coding could have generated $£ 473$ more income, most notably by correctly specifying the type of operation for common procedures.

Following our first cycle, we discussed the findings with our clinical coding team in order to address the sources of error. We were able to address misinterpretation of clinical terms which had led to inaccurate coding. Another source of error was the quality of the recorded data regarding the exact nature of the surgical procedure. To address this, we also created a coding proforma in collaboration with other relevant clinicians, theatre staff and coding staff. The operating surgeon subsequently filled in the proforma in theatre for every oculoplastic case during the re-audit cycle. Our re-audit demonstrated an improvement in coding accuracy.

\section{Comparison with literature}

Our high rate of coding error is comparable with papers in the literature, although there is wide variation in the reported rates. Burns et al. [11] found an overall median accuracy of $83.2 \%$, with variation between studies ranging from 50.5 to $97.8 \%$.

Subsequent papers also report large variations across the surgical specialties. Haliasos et al. [3] found at least one coding error in $18.4 \%$ of neurosurgical cases, and HRG changes in $10.4 \%$, with a loss of $£ 111$ per patient episode. In Nouraei et al. [13], their ENT coding audit led to at least one change of coding in $44 \%$ of cases, and to HRG changes in $16 \%$, with an income variance of $£ 109.46$ per patient. In Naran et al. [14], their audit led to at least one change of coding in $77 \%$ patients, and to HRG code changes in $54 \%$ patients, with an estimated $£ 114$ in lost revenue per patient. In Martinou et al. [15], 32\% of their breast and endocrine operations were incorrectly recorded with an estimated loss of $£ 17,000$ to the Trust over the 2-month study period. Cheema and Khwaja [16] had an error of $20 \%$ in their audit of urology cases, with revised $\mathrm{HRG}$ codes leading to a recovery of $£ 10,716$.

Murphy et al. [17] found that $14.5 \%$ of primary procedures were incorrect and $37.6 \%$ had additional procedures that were not recorded. As with our audit, the authors introduced a coding proforma that was completed in theatre and found a $7.2 \%$ non-significant improvement in accuracy of coding primary procedures and a $21 \%$ significant improvement in accuracy of coding additional procedures $(P<0.001)$. 
Our results are also comparable with Nouraei et al. [12]. Overall, the incidence of HRG change across Surgery fell significantly from $22 \%$ in their first audit cycle to $5.8 \%$ in the second cycle $(P<0.0001)$.

\section{Understanding the cause of coding errors}

Coding errors can arise from multiple sources, and it is challenging to definitively identify the causes. Nouraei et al. [12] classifies the causes by clinician, coder, system, language, case-notes, and process factors. Although this is not an exhaustive list, it is valuable in targeting areas for improvement.

Clinician factors include lack of awareness of coding issues and diagnostic uncertainty [12, 14]. Clinicians have little training and minimal exposure to the complexities of coding. Furthermore, illegible handwriting, use of abbreviations, and short or incompletely descriptive handwritten notes can affect accurate coding. This is particularly important in Trusts where operation notes are still being recorded by hand and not electronically. Arthur and Nair [18] found that procedures with type-written notes were accurately recorded in $97 \%$ of cases versus $85 \%$ of those with handwritten notes. They suggest using template-based mandatory fields in typed notes as a prompt for generating complete notes.

Coder factors include recruitment, experience, specialisation and time-pressure [12, 14]. Colville et al. [19] and Tatham and Castillo [20] found that clinical activity is more likely to be coded accurately if done by the clinical coding department than if the procedure is coded directly in theatre. However, Kim et al. [21] demonstrated that coding with specialist clinical terms is strongly related to the knowledge of the field and complexity of the terms. As seen in our audit, confusion about muscle nomenclature in the operation notes can complicate accurate coding. Accurate documentation is important and any conflicting information is confusing for coders who are not necessarily ophthalmically trained.

\section{Addressing the underlying causes}

These multifactorial causes, once identified, can be addressed in multiple ways. Firstly, educating clinicians and coders has been shown to significantly reduce coding inaccuracies. Following education, Nouraei et al. [13] found HRG changes reduced significantly from $16 \%$ of cases to 9\% $(P<0.001)$ in their re-audit. Cheema and Khwaja [16] found reduced clinical coding error from $20 \%$ of cases to $11 \%$ in their re-audit.

Secondly, continuous interaction between clinicians and clinical coders can help minimise misunderstandings or confusion. Pillai and Medford [22] demonstrated a $100 \%$ coding accuracy rate for endobronchial ultrasound-guided transbronchial needle aspiration where the physicians engaged with the coders to prevent errors. Yeoh and Davies [23] found increased coding accuracy from 54 to $85 \%$. Nouraei et al. [2] showed that involving clinicians in a multi-disciplinary coding team led to $24.1 \%$ of records being changed with an increase in income of $£ 443,371$ to their department, and they suggest that clinician involvement may be a cost-effective way of improving reimbursement.

Thirdly, agreeing a coding procedure between clinicians and coder can help reduce coding errors. This can be achieved, for example, through the use of coding proformas, and the implementation of quality assurance programmes such as regular internal audit $[11,14,24]$.

\section{Implications}

Clinical codes provide a method of tracking and retrieving records of procedures. This is particularly important in an increasingly data-driven NHS [25]. Apart from the financial aspects already discussed, access to accurate records of a department's performance is necessary for clinical audit, allocation of resources and for health service planning. Coding inaccuracies can falsely simplify cases that consume resources, leading to under-recovery of reimbursement, or give the wrong picture of a department's workload [3].

Extra clinical details and more accurate coding did not necessarily lead to significant changes in HRG codes and subsequent reimbursement for the majority of our patients. The main reason is probably that the PbR tariffs do not always reflect the complexity of procedures, a fact that was also noted by Tatham and Castillo [20]. Additionally, clinical codes provide more than just a method of reimbursement. It is also a main method of retrieving records of procedures and reflecting on the department's activity. As tariffs become further individualised, or future changes occur to payment contracts, greater coding detail can allow better and more accurate reimbursement of actual clinical activity [20].

Finally, it is worth noting that Nouraei et al. [12] argue that although it is possible to reduce coding errors and improve clinical coding accuracy by more than $40 \%$ $[12,26]$, it is impossible to eliminate coding errors altogether, as there will always be cases with diagnostic uncertainty or variation in interpretation.

\section{Strengths and limitations of our study}

We have designed a simple and robust system that can be applied realistically during busy theatre lists in view of its ease of completion. The strengths of our study include a consecutive evaluation of all procedural coding in both 
study cycles with the support of our coding and clinical colleagues. The proforma was designed on the basis of feedback from all parties. This has been widely disseminated and adopted by all oculoplastic surgeons in our department. Our study demonstrated how the proforma has improved our coding accuracy rates. We also demonstrated that increased communication and engagement with the clinical coding department can improve all round understanding of the coding process, which is especially useful in the more complex procedures.

However, we recognise that coding is an intricate system which is open to interpretation. In our endeavours to standardise the coding proforma, we had to exclude less commonly performed procedures, which arguably may need more input from clinicians to aid the coders than common procedures that the coders may be more accustomed to. However, our project has demonstrated that even the commonly coded procedures can require additional help for the coders.

\section{Conclusions}

Our study shows that clinical coding is a complex area that is subject to inaccuracies. This has financial implications for hospital trusts.

All healthcare professionals involved in the patient's journey have a role in ensuring accurate coding. We have implemented a simple but robust system that has improved coding accuracy and reduced the loss of income. As a consequence of this work we have established stronger links with our coders and plan to extend our audit into other clinical areas. Coding variability may be impossible to eliminate completely, but it can be improved through education and quality assurance programmes, involving more input from the surgical team and through better communication between clinicians and the coding team.

The proforma can be easily adapted to other ophthalmic subspecialties, as well as other surgical specialties, which could have significant financial implications for individual Trusts and the NHS as a whole.

\section{Summary}

\section{What was known before}

- Reimbursement for clinical activity depends on accurate coding.

- Coding is complex and the final tariff is dependent on multiple factors.

- Coding errors can lead to an inaccurate picture of clinical activities with potential financial implications.

\section{What this study adds}

- Accuracy of clinical coding of surgical interventions depends on high-quality data.

- Increased engagement with the coding department and use of a surgical coding proforma improved coding accuracy with financial benefits.

\section{Compliance with ethical standards}

Conflict of interest The authors declare that they have no conflict of interest.

Publisher's note: Springer Nature remains neutral with regard to jurisdictional claims in published maps and institutional affiliations.

\section{References}

1. Audit Commission Archives. The right result? Payment by Results 2003-07. London: Audit Commission. http://weba rchive.nationalarchives.gov.uk/20080527103909/. http://www. audit410commission.gov.uk/reports/NATIONAL411REPORT. asp?CategoryID $=$ ENGLISH^574\&ProdID $=30321654-7$ A784be6-412ADA3-C2FC1AD3B515. Accessed 26 Feb 2018.

2. Nouraei SA, O'Hanlon S, Butler CR, Hadovsky A, Donald E, Benjamin E, et al. A multidisciplinary audit of clinical coding accuracy in otolaryngology: financial, managerial and clinical governance considerations under payment-by-results. Clin Otolaryngol. 2009;34:43-51.

3. Haliasos N, Rezajooi K, O’neill KS, Van Dellen J, Hudovsky A, Nouraei S. Financial and clinical governance implications of clinical coding accuracy in neurosurgery: a multidisciplinary audit. Br J Neurosurg. 2010;24:191-5.

4. World Health Organization. International statistical classification of diseases and related health problems (ICD-10). 10th ed. Geneva: World Health Organization; 1994.

5. Connecting for Health, The Stationery Office. OPCS classification of interventions and procedures Version 4.4. Norwich: Connecting for Health, The Stationery Office; 2007.

6. HRG Grouper. 2008. https://digital.nhs.uk/National-casemixoffice/downloads-groupers-and-tools.

7. Coatesworth A. Payment by Results? BAO-HNS April. 2007; 17:2.

8. The King's Fund. Anon. Payment by Results. April 2005.

9. Mitra I, Malik T, Homer JJ, Loughran S. Audit of clinical coding of major head and neck operations. Ann R Coll Surg Engl. 2009;91:245-8.

10. NHS Improvement. Reference costs 2016/17: highlights, analysis and introduction to the data. November 2017. https://improvement. nhs.uk/resources/reference-costs/. Accessed 26 Mar 2018.

11. Burns EM, Rigby E, Mamidanna R, Bottle A, Aylin P, Ziprin P, et al. Systematic review of discharge coding accuracy. J Public Health (Oxf). 2012;34:138-48.

12. Nouraei SA, Hudovsky A, Frampton AE, Mufti U, White NB, Wathen CG, et al. A study of clinical coding accuracy in surgery: implications for the use of administrative big data for outcomes management. Ann Surg. 2015;261:1096-107.

13. Nouraei SA, Hudovsky A, Virk JS, Chatrath P, Sandhu GS. An audit of the nature and impact of clinical coding subjectivity 
variability and error in otolaryngology. Clin Otolaryngol. 2013; 38:512-24.

14. Naran S, Hudovsky A, Antscherl J, Howells S, Nouraei SA. Audit of accuracy of clinical coding in oral surgery. Br J Oral Maxillofac Surg. 2014;52:735-9.

15. Martinou E, Shouls G, Betambeau N. Improving the accuracy of operation coding in surgical discharge summaries. BMJ Qual Improv Rep. 2014;3:u202053.w1990

16. Cheema ZA, Khwaja SA. Implications of miscoding urological procedures in an era of financial austerity- 'Every Penny Counts'. JRSM Open. 2015;6:2054270415593463.

17. Murphy J, May C, Di Carlo S, Beckingham I, Cameron IC, Gomez D Coding in surgery: impact of a specialized coding proforma in hepato-pancreato-biliary surgery. ANZ J Surg. 2017. https://doi.org/10.1111/ans.14076

18. Arthur J, Nair R. Increasing the accuracy of operative coding. Ann R Coll Surg Engl. 2004;86:210-2.

19. Colville RJ, Laing JH, Murison MS. Coding plastic surgery operations: an audit of performance using OPCS-4. Br J Plast Surg. 2000;53:420-2.
20. Tatham A, Castillo A. Clinical coding of surgical procedures in the ophthalmology department. Eye (Lond). 2010;24:182-5.

21. Kim SY, Kim HH, Shin KH, Kim HS, Lee JI, Choi BK. Comparison of knowledge levels required for SNOMED CT coding of diagnosis and operation names in clinical records. Health Inf Res. 2012;18:186-90.

22. Pillai A, Medford AR. Greater physician involvement improves coding outcomes in endobronchial ultrasound-guided transbronchial needle aspiration procedures. Respiration. 2013; 85:417-21.

23. Yeoh C, Davies H. Clinical coding: completeness and accuracy when doctors take it on. Brit Med J. 1993;306:972.

24. O'Dowd A. Coding errors in NHS cause up to $£ 1$ bn worth of inaccurate payments. Brit Med J. 2010;341:c4734.

25. Darzi A. Evidence-based medicine and the NHS: a commentary. JRSM. 2008;101:342-4.

26. To T, Estrabillo E, Wang C, et al. Examining intra-rater and interrater response agreement: a medical chart abstraction study of a community-based asthma care program. BMC Med Res Methodol. 2008;8:29. 